\title{
Calculation of the electrodynamic forces causing deformation of the power transformer's windings
}

\author{
A $Y u$ Khrennikov ${ }^{1, *}, N M$ Aleksandrov ${ }^{2, *}$ \\ ${ }^{1}$ Moscow, Russia \\ ${ }^{2}$ Cheboksary, Russia
}

\begin{abstract}
This paper describes modified method to calculate electrodynamic forces causing deformation of the power transformer's windings. This method takes into account the deformed shape of the winding. The new allow to get better accuracy.
\end{abstract}

\section{Introduction}

Power transformers are one of the most important devices being used at the transmission and distribution of electricity. Mechanical damage to the windings of transformers lead to their failure and, accordingly, cause interruptions in power supply. Mechanical damage is mainly the result of electromagnetic forces acting on the transformer windings during short circuits [1]. Improper transportation of the power transformer or damage of the on-load tap-changer can also cause various mechanical deformations. In case of significant deformations, subsequent internal electrical damage is possible when it is turned on. Repair of such a transformer requires considerable time and effort, as well as high material costs due to the time they are taken out of work and the repair of power transformers themselves. Thus, the diagnosis of mechanical damage before commissioning will help to avoid negative consequences in the event of subsequent rapid and less expensive overhaul.

The reliability of power supply, the quality of electricity and its economic value depend on the state of the transformer. Damage of power transformers can have a serious negative effect on the environment, such as a fire or spill of transformer oil. Consequently, accidents in power transformers are of particular concern and are thoroughly investigated.

Errors in the calculations and insufficient provision of mechanical strength (in terms of explosion protection from short-circuit currents) can cause serious damage to the transformer, for example,

- Deformation of the low-voltage or high-voltage winding;

- Destruction and curvature of winding pressing elements;

- Damage of the transformer tank;

- Destruction of high-voltage bushings;

- etc.
Development of systems for assessing and monitoring the state of the windings of power transformers will reduce costs throughout the life cycle of the transformer and ensure reliable operation of power transformers.

Almost any external damage of a power transformer is fairly easy to detect visually. Damage to the active part of the transformer requires opening the transformer for internal inspection. Often such interference is undesirable because of the possibility of moisture entering the transformer.

Reliable determination of mechanical damage in power transformers due to deformation of the windings requires the use of a sensitive method for determining this type of damage. Some of the diagnostic methods used for this purpose are:

\section{Mathematical model and the equation of forces for radial deformation}

To obtain a mathematical model of deformation, it is necessary to develop a model of geometric changes in the winding and, on its basis, derive an equation for electrodynamic forces acting on the winding.

This section describes a mathematical model of the radial deformation of a single turn of a transformer winding in the form of an equation; it also shows a figure (Figure 1) depicting this deformation. The mathematical model of the radial deformation of one turn of the transformer winding in the form of an equation is more accurate than the previously presented models. Due to the use of trigonometric functions when describing the shape and volume of deformation and smaller error, this model more realistically reflects the processes occurring in the windings of power transformers in the case of short circuit currents.

Assumptions adopted in the preparation of the model:

- the maximum size of the convexity is assumed to be equal to the size of the concavity: a,

\footnotetext{
* Corresponding author: nickdynamics@ gmail.com
} 
- the convexity range is assumed to be equal to the concavity range: angle $\Phi / 2$.

As an equation describing the concave-convex deformation of the turn of the conductor, the function $\varepsilon$ $(\phi)$ is chosen, described in polar coordinates (1):

$$
\varepsilon(\phi)=\mathrm{a} \cdot \sin (2 \cdot \pi \cdot \phi / \Phi), \text { при } 0 \leq \phi<\Phi,
$$

where $\mathrm{a}$ is the maximum deformation - the maximum deviation of the distorted section from the ideal state, $\Phi$ the deformation range - the central angle within which the deformation is observed, $\phi$ is the function argument, the angle that is set clockwise from the vertical semiaxis.

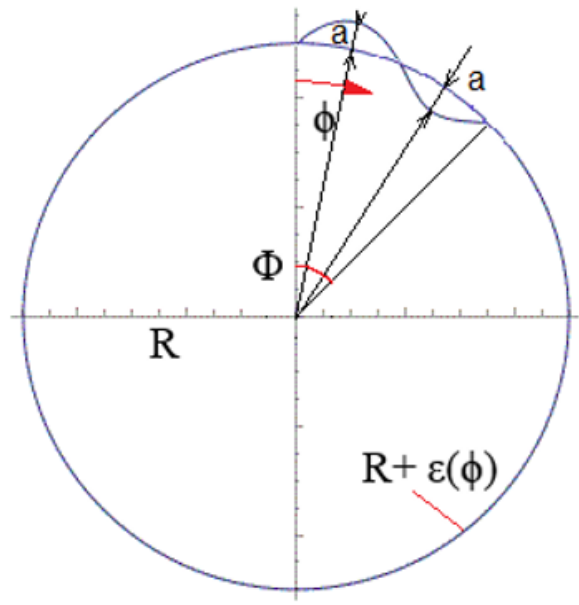

Fig. 1. Winding coil deformation model.

Radial forces stretch the external winding, seeking to break it, and compress the internal [2]. To calculate the forces acting on the winding, it is customary to use either the Bio-Savart law (1) or the Lagrange theorem (2).

$$
F=\int_{V}[B j] d V
$$

In this case, we use the Lagrange theorem to calculate the electromagnetic forces during radial deformation. When applied to an electromagnetic field, the theorem is formulated as follows: in a system of circuits with a current in a magnetic field, an electromagnetic force that tends to change a given coordinate of the system is equal to the derivative of the magnetic field energy from this coordinate under the assumption that the currents in the circuits are kept constant [2]

$$
f_{g}=\frac{d W_{M}}{d g}
$$

where $f_{g}$ is the force acting in the direction of the coordinate $\mathrm{g} ; \mathrm{W}_{\mathrm{M}}$ is the energy of the transformer's magnetic field.

$$
W_{M}=\frac{1}{2} \mu_{0} \int_{V} B_{\sigma}^{2} d V
$$

$$
W_{M}=\frac{1}{2} L_{k} i^{2}
$$

where $\mathrm{L}_{\mathrm{k}}$ - short circuit inductance of the transformer.

Thus,

$$
f_{g}=\frac{1}{\mu_{0}} \int_{V} B_{\sigma}^{2} \frac{\partial B_{\sigma}}{\partial g} d V=\frac{1}{2} i^{2} \frac{\partial L_{K}}{\partial g}
$$

Radial forces acting on the windings of two winding transformers tend to increase the distance between the windings $\delta_{12}$ (figure 2).

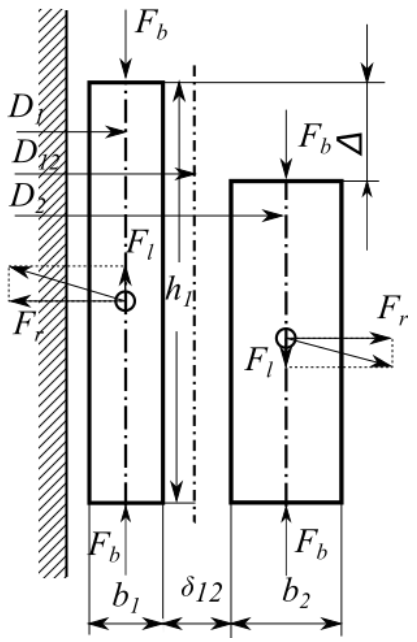

Fig. 2. Radial forces acting on the windings of a power transformer.

Therefore, in accordance with equation (5), it is necessary to take the derivative with respect to $\delta_{12}$.

$$
F_{r}=\frac{1}{2} I_{\max }^{2} \frac{\partial L_{K}}{\partial \delta_{12}}
$$

The short circuit inductance is calculated by the formula

$$
L_{K}=\frac{\mu_{0} \omega^{2} \rho_{1} \pi D_{12}\left(\delta_{12}+\frac{b_{1}+b_{2}}{3}\right)}{h}
$$

Knowing that the shape of the coil winding, according to the above model (figure 2), varies according to equation (1), the radial force acting on the winding is calculated by the formula

$$
F_{r}=\frac{\mu_{0}\left(I_{\max } \omega\right)^{2} \rho_{1} \pi\left(D_{12}+a \cdot \sin (2 \pi \varphi / \Phi)\right.}{2 h}
$$

where $F_{r}$ - is the radial force; $\mu_{0}-$ magnetic conductivity; Imax - short circuit current amplitude; $\omega$ - the number of turns of the winding; $\rho_{1}-$ is the coefficient of bringing the ideal stray field to the real one (Rogowski coefficient); $\mathrm{D}_{12}$ is the distance of the tank wall to the 
middle of the gap between the windings; $h$ - is the height of the winding.

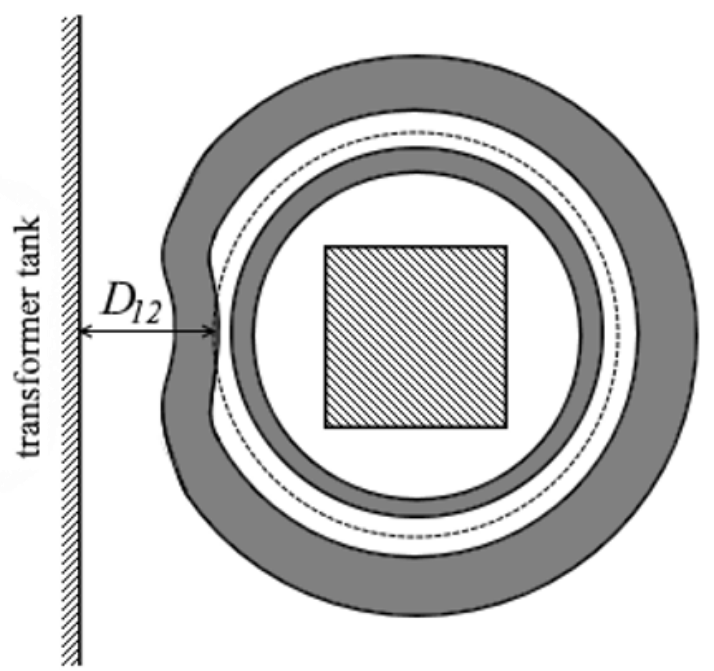

Fig. 3. Changing the shape of the winding under the action of radial forces.

Equation (7) for the radial force more accurately reflects the processes occurring in the windings of power transformers in case of short circuit and the occurrence of radial forced bending due to the use of trigonometric functions in describing the shape and volume of deformation.

\section{Mathematical model and equation of forces for elliptic deformation}

In some [Marianna] publications, a theoretical case is considered when a coil (or segment) of a winding of radius $\mathrm{R}$ (equal to the inner radius $\mathrm{R} 1$ or outer radius $\mathrm{R} 2$ according) located between the magnetic core and the tank wall is slightly deformed, and its shape is changed and does not a circle, and an ellipse (figure 4). transformer tank

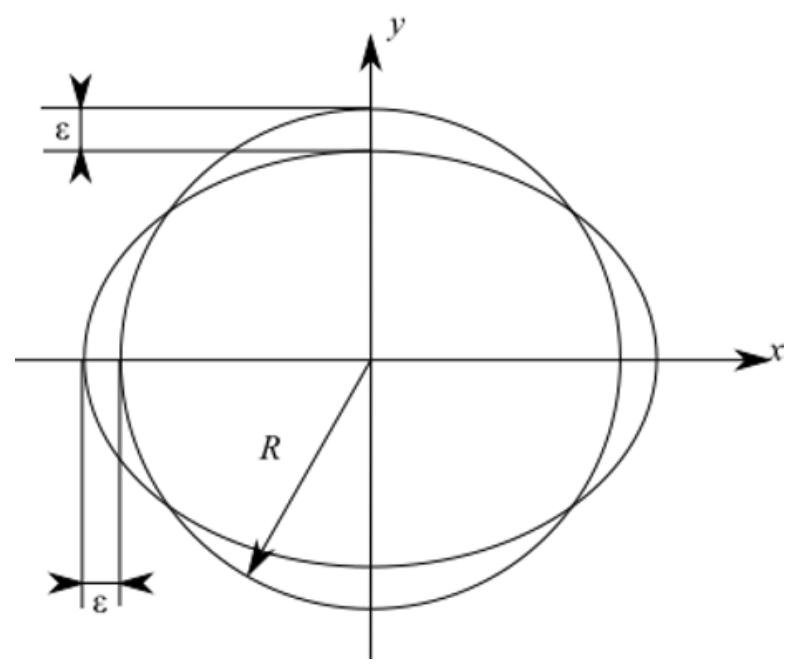

Fig. 4. Elliptical deformation of the winding.
The equation of geometric deformation in the first approximation for small values of the ratio $\varepsilon / \mathrm{R}$ is as follows

$$
\delta(r, \varphi)=r-R=\varepsilon \cdot \cos (2 \varphi)
$$

where $\mathrm{R}$ - is the radius of the not deformed winding, $\mathrm{r}$ is the curve describing the elliptical deformation, $\varepsilon=R$ $r\left(90^{\circ}\right)$.

At small values of the ratio $\varepsilon / R$, the condition of maintaining the length of the coil before and after deformation is satisfied:

$$
l=2 \pi R\left(1+\frac{\varepsilon^{2}}{4 R^{2}}\right) \approx 2 \pi R
$$

Substituting the equation of elliptic deformation into the force equation for radial deformation, we get

$$
F_{r}=\frac{\mu_{0}\left(I_{\text {макс }} \omega\right)^{2} \rho_{1} \pi\left(D_{12}+\varepsilon \cdot \cos (2 \varphi)\right)}{2 h}
$$

\section{Conclusion}

In this paper the modified method was presented to calculate electrodynamic forces causing deformation of the power transformer's windings. This method takes into account the deformed shape of the winding. The new equations allow to get better accuracy and will help in transformer's design

\section{References}

1. A.Yu. Khrennikov, Power transformer's fault diagnostics at SAMARAENERGO Co, including FRA/LVI method, Reports from School of Math. and System Engineering, Vaxjo University, Sweden, 43 (2000) ISSN: 1400-1942.

2. S.B. Vasutinskiy, Calculation and design of transformers (1976)

3. R.P.P. Smeets, L.H.T. Paske, P.P. Leufkens, T. Fogelberg, Thirteen Years Test Experience With Short-Circuit Withstand Capability of Large Power Transformers, presented at the CIGRE 6th Southern Africe Regional Conference (2009)

4. T. Fogelberg, Surviving a short-circuit, Behind the plug, 24 (2008)

5. E. Betancourt, C. Hernandez, Power Transformer Manufacturer's Experience with Transportation Mishandling, 7th Annual WEIDMANN Diagnostic Solutions Technical Conference (2008)

6. A. Kraetge, M. Kruger, P. Fong, Frequency response analysis - status of the worldwide standardization activities, in International Conference on Condition Monitoring and Diagnosis, 651-654 (2008)

7. E.P. Dick, C.C. Erven, Transformer Diagnostic Testing by Frequency Response Analysis, IEEE 
Transactions on Power Apparatus and Systems, PAS-97, 2144-2153 (1978)

8. M. Wang, A.J. Vandermaar, K.D. Srivastava, Review of condition assessment of power transformers in service, IEEE Electrical Insulation Magazine, 18, 12-25 (2002)

9. P. Picher, J. Lapworth, T. Noonan, J. Christian, Mechanical condition assessment of transformer windings using frequency response analysis, Technical Brochure, 342 (2008)

10. Z.W. Zhang, W.H. Tang, T.Y. Ji, Q.H. Wu, FiniteElement Modeling for Analysis of Radial Deformations Within Transformer Windings 